\title{
Effect of Carbon Tetrachloride (CCL4) on Liver in Adult Albino Rats: Histological study
}

Haytham EL Sayed Ali EL Sayed ${ }^{1}$, Lotfy EL Sayed Morsy ${ }^{1}$, Tamer Mosad Abo Emara ${ }^{1}$, Rania A. Galhom ${ }^{2}$

${ }^{1}$ Histology and Cell Biology Department, Faculty of Medicine, Al-Azhar University, Cairo, Egypt. ${ }^{2}$ Human Anatomy and Embryology Department, Faculty of Medicine, Suez Canal University, Esmaillia, Egypt.

\begin{abstract}
Background: The liver is a vital organ that plays a key role in the detoxification of endogenous and exogenous substances. Variety of pathological factors including viral hepatitis (especially hepatitis B and C), alcohol and drug abuse, metabolic diseases, autoimmune diseases and congenital abnormalities can cause hepatic injury. Chronic hepatic disease is quite common in daily clinical practice. Liver cirrhosis is the final stage of all chronic hepatic diseases. Liver transplantation is the treatment of choice for chronic liver failure, although, it faces several difficulties.
\end{abstract}

Aim of the work: to evaluate the toxic effect of carbon tetrachloride $\left(\mathrm{CCL}_{4}\right)$ on the liver structure.

Materials and methods: Twenty male rats of average weight 200 grams and aged 6-8 weeks were included in this study. The Rats were divided into the following two groups: Group I (control group): ten male rats were subdivided into two subgroups, one of them acted as negative control and the other received the equivalent volume of the solvent olive oil. Group II (experimental group): ten male rats were injected intraperitoneally (IP) by $\mathrm{CCL}_{4}$ twice weekly for four weeks.

Results: Histological examination of liver specimens showed that $\mathrm{CCl}_{4}$ caused patchy and variable pathological changes in the liver tissue along with significant increase in collagen fiber content.

Conclusion: The induced liver fibrosis by $\mathrm{CCL}_{4}$ in rats showed markedly pathological effects on hepatic stroma and parenchyma.

Keywords: Carbon Tetrachloride CCL4, Liver, Albino Rats

\section{INTRODUCTION}

Carbon tetrachloride is the organic compound with the formula CCL4. It was originally synthesized by the French chemist Henri Victor Regnault in 1839 by the reaction of chloroform with chlorine, but now it is mainly produced from methane. It was formerly widely used in fire extinguishers, as a precursor to refrigerants and as a cleaning agent ${ }^{(\mathbf{1})}$.

$\mathrm{CCL}_{4}$ does not occur naturally, it is a clear liquid with sweet smell that can be detected at low levels ${ }^{(2)}$. $\mathrm{CCL}_{4}$ is a common environmental pollutant. Workers are at high risk of exposure to high levels through inhalation and dermal contact. On the other hand, the general population may be exposed to low levels of $\mathrm{CCL}_{4}$ through inhalation from the atmospheric environment ${ }^{(3)}$.

Because of its harmful effects, its uses are now banned and it is only used in some industrial applications. The primary routes of potential human exposure to $\mathrm{CCL}_{4}$ are inhalation, ingestion and dermal contact $^{(4)}$.

Weber et al. ${ }^{(5)}$ mentioned that $\mathrm{CCL}_{4}$ is also well known for hepatic toxic actions. They reported that it causes acute liver damage like necrosis and steatosis. This effect is due to the release of free radicals which are composed of trichloromethyl $\left(\mathrm{CCL}_{3}\right)$ and peroxy trichloromethyl $\left(\mathrm{OOCCL}_{3}\right)$ radicals. These free radicals can generate lipid peroxide which may cause cell membrane damage, alteration in enzyme activity and finally induction of hepatic injury and necrosis.
The mechanism of $\mathrm{CCL}_{4}$ induced hepatotoxicity, especially necrosis and fatty liver, has long been a challenging subject of many researchers from various fields over the past 50 years. Even though the mechanisms of tissue damages are different among chemicals and affected tissues, $\mathrm{CCL}_{4}$ played a role as a key substance of tissue injury. A number of studies were conducted and various hypotheses were raised. As a result, several important basic mechanisms of tissue damages were emerged, involving metabolic activation, reactive free radical metabolites, lipid peroxidation, covalent binding and disturbance of calcium homeostasis ${ }^{(\boldsymbol{6})}$.

Liver is an important organ which plays a central role in metabolic homeostasis. It also has an amazing regenerative capability after liver mass loss (7). Iredale et al. ${ }^{(8)}$ described liver fibrosis as a pathological condition characterized by an excessive deposition of collagen and other components of ECM. They added that the liver fibrosis was originally considered progressive and irreversible, but several clinical studies had shown that it could be reversed if the causative agents were adequately removed or if patients were treated effectively.

Hepatic fibrosis was historically thought to be a passive and irreversible process due to the collapse of the hepatic parenchyma and its substitution with a collagen-rich tissue. Currently, it is considered to be a model of the wound healing response to chronic liver injury ${ }^{(9)}$. 
Guyot et al. ${ }^{(10)}$ explained the causes of hepatic fibrosis. They concluded that fibrosis is a common pathological process resulted from various chronic hepatic injuries such as viral hepatitis, alcohol consumption, autoimmune disorders, drug-induced hepatitis, helminthic infection, iron or copper overload and biliary obstruction.

\section{AIM OF THE WORK}

The aim of the current research was to assess the toxic effect of the CCL4 on the liver of adult male albino rats.

\section{MATERIALS AND METHODS}

\section{A. Animal model:}

Twenty albino rats of average weight $200 \mathrm{gm}$ and aged 6-8 weeks were included in this study. The animals were purchased and raised in The Faculty of Medicine, Al-Azhar University. They were housed in plastic cages with mesh wire covers and were given food and water ad libitum.

B. Materials:

- Carbon tetrachloride $\left(\mathrm{CCL}_{4}\right)$ of $100 \%$ concentration.

- Olive oil. Both were obtained from Algomhoria Company.

- Phosphate buffer saline (PBS): 0.0067 M (PO4) without $\mathrm{Ca}^{+2}$ and $\mathrm{Mg}$ sterile filtered for cell culture. It was purchased from Lonza Company, Swiss.

C. Dose:

Carbon tetra chloride dissolved in olive oil was given by intraperitoneal (IP) injection in a dose of $0.5 \mathrm{mg} / \mathrm{Kg}$ body weight of rat twice weekly ${ }^{(8)}$.

D. Preparation of the solution:

$100 \mathrm{mgs}$ of $\mathrm{CCL}_{4}$ were dissolved in $100 \mathrm{mLs}$ of olive oil. So each rat was given $0.1 \mathrm{~mL}$ of the prepared solution intraperitoneally twice weekly.

\section{The animals were classified into two main groups:}

Group I (control group): Ten albino rats (6-8 weeks) with average weight $200 \mathrm{gm}$ were further subdivided into two subgroups:

- Subgroup IA (5 rats): included rats which received nothing. They were used to collect liver specimen as a negative control group.

- Subgroup IB (5 rats): Each rat was given equivalent volume $(0.1 \mathrm{~mL})$ of olive oil by IP injection twice weekly for four weeks then the rats were sacrificed ${ }^{(11)}$. Group II (experimental group): included 10 albino rats (6-8 weeks) with average weight $200 \mathrm{gm}$. Rats were subjected to IP injection of $\mathrm{CCL}_{4}$ dissolved in olive oil (in a dose of $0.5 \mathrm{mg} / \mathrm{Kg}$ ) twice weekly for four weeks then sacrificed ${ }^{(8)}$.

Ethical approval and written informed consent: An approval of the study was obtained from AlAzhar University academic and ethical committee. This study was conducted in accordance with ethical procedures and policies approved by Animal Care and Use Committee of Faculty of Medicine, Al-Azhar University, Cairo, Egypt.

\section{Preparation of liver specimens for light microscopic examination}

The liver specimens were taken from the right lobe. They were fixed in buffered formalin for one week and then they were dehydrated in ascending grades of alcohol. Then the specimens were cleared in xylene; two changes half an hour each. After that they were embedded in two changes of soft paraffin, one hour each at oven $58^{\circ} \mathrm{C}$. Then paraffin blocks were obtained, serial sections of $5 \mu \mathrm{m}$ thickness were cut and stained with the following histological stains.

1. Haematoxylin and Eosin ( $\mathrm{H}$ and $\mathrm{E})$.

2. Masson's trichrome stain.

3.

\section{Statistical analysis:}

The statistical package for the social sciences (SPSS) computer program; version 20 was used. Data are presented as means+SD. Student t-test was used to compare the collagen area percentage in the 2 main groups.

\section{RESULTS \\ The Light microscopic results Group I (control group)}

\section{A. Histological results:}

The control groups (Subgroups IA and IB) showed no histological or morphometric difference between them. Examination of $\mathrm{H}$ and $\mathrm{E}$ stained sections of all control rats (group I) revealed that they showed nearly the same histological picture. The liver appeared to be composed of ill-defined hexagonal classic lobules. Each lobule was traversed centrally by a central vein. The parenchyma of these lobules was composed of liver cells (hepatocytes). The hepatocytes appeared to be arranged in the form of branching cords that radiating from the central veins to the periphery of the classic hepatic lobules (Fig. 1).

These cords were separated by blood sinusoids, which were lined by flat endothelial cells. The hepatocytes showed acidophilic cytoplasm with single central rounded vesicular nuclei and some of the cells were binucleated.

At the periphery of the classic hepatic lobules, portal areas were noticed. The portal area was composed of branches of hepatic artery, portal vein and bile duct (Fig. 2).

In Masson's trichrome stained sections the parenchyma of the liver appeared to be supported with a stroma of very delicate meshwork of collagenous fibers. Ill-defined septa were seen in-between the classic hepatic lobules and in the capsule (Fig. 3). Few collagenous fibers were well seen surrounding the central veins and the structures in the portal areas (Fig. $4)$. 


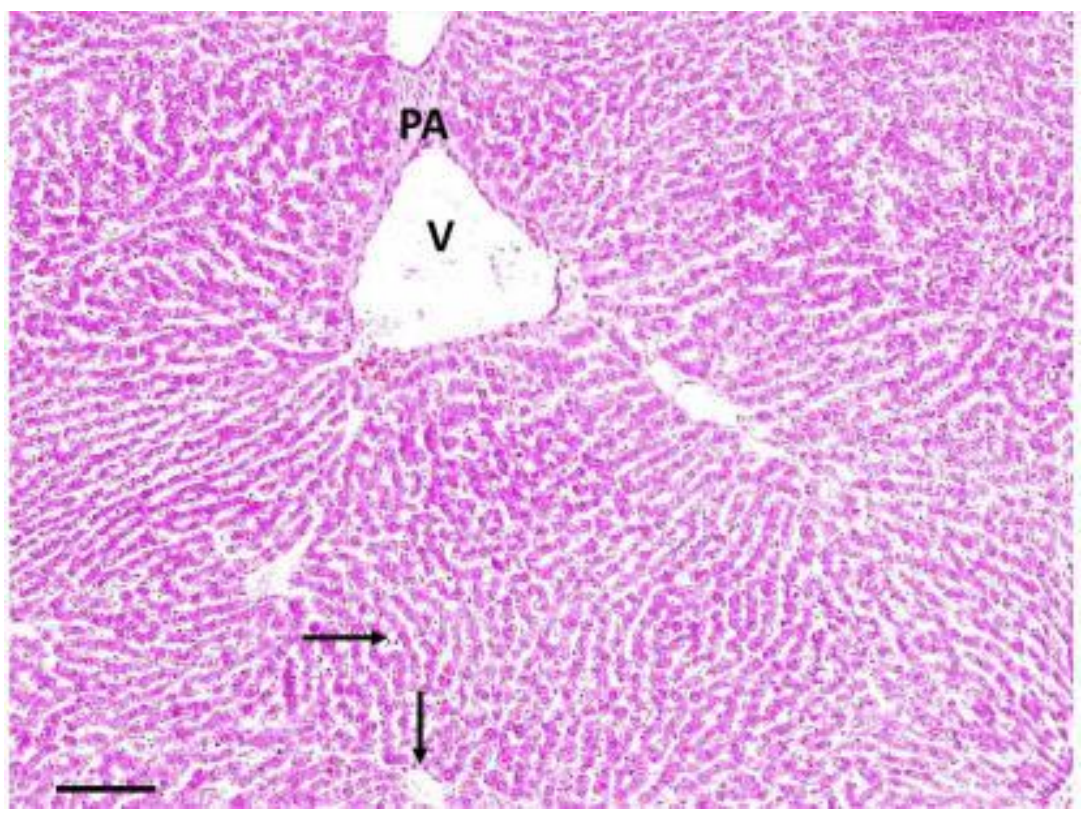

Fig. (1): A photomicrograph of a liver section of a control rat showing the general appearance of the hepatic lobules. Notice the central vein $(\downarrow)$ and the peripherally situated portal area (PA) with branches of portal vein (V). H and $\mathrm{E}$ X100

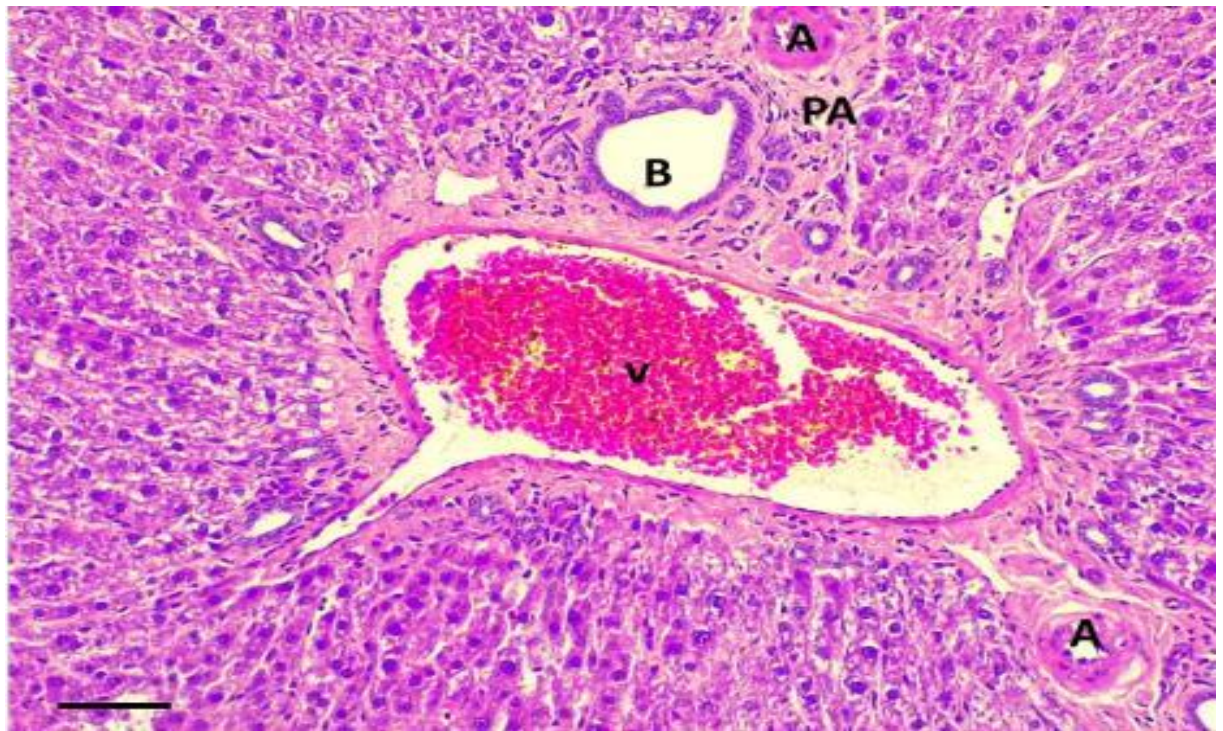

Fig. (2): A photomicrograph of a liver section of a control rat, showing the portal area $(\mathrm{P})$ with branch of portal vein (V), hepatic artery (A) and a branch of bile duct (B). H and E X200

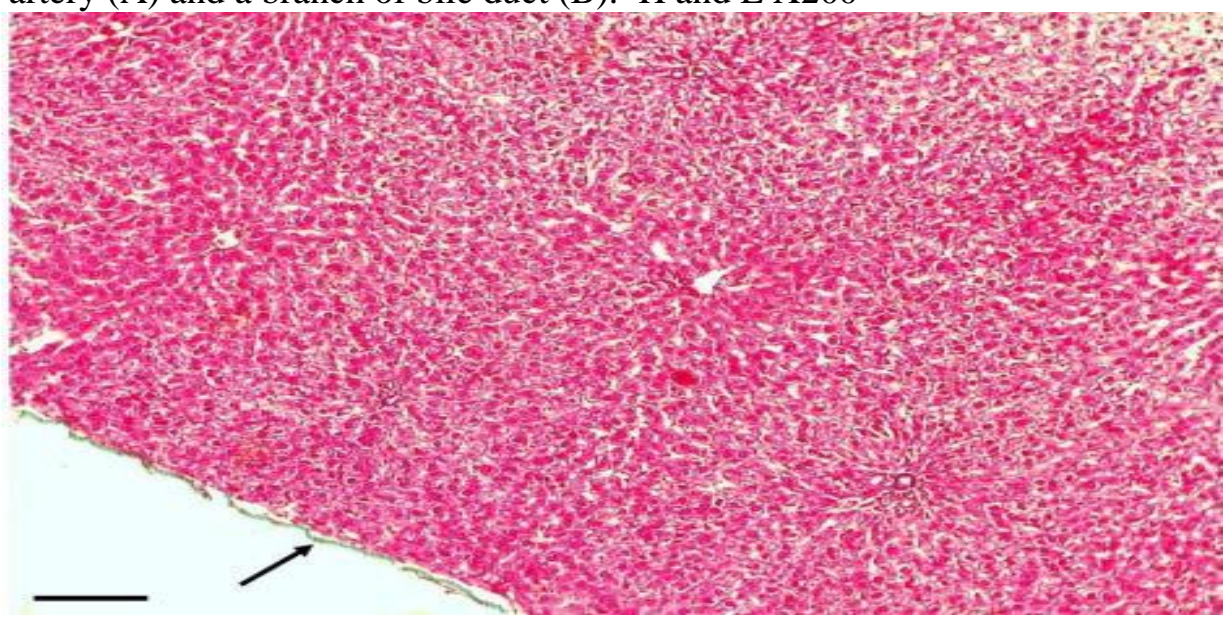

Fig. (3): A photomicrograph of a liver section of a control rat, showing ill-defined septa in-between the classic hepatic lobules and few collagenous fibers within the capsule (arrow) Masson's trichrome stain X 100 


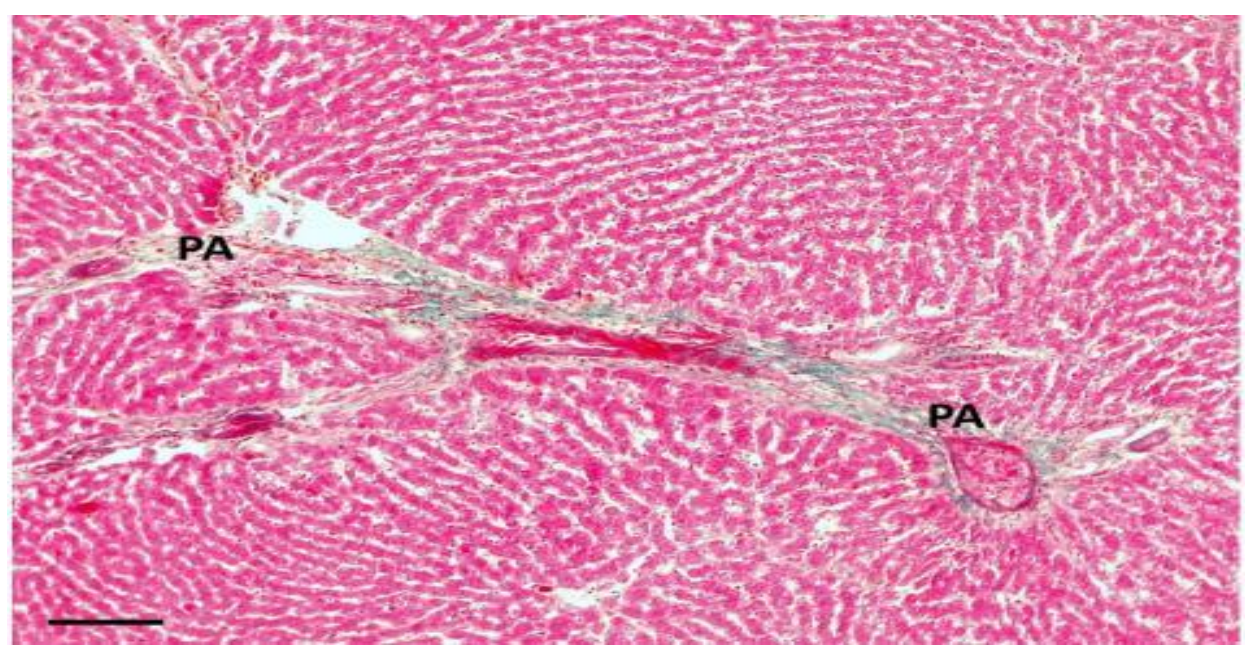

Fig. (4): Photomicrographs of a liver section of a control rat, showing few collagen fibers $(\uparrow)$ around the structures of the portal area (PA) Masson's trichrome stain X 200

\section{Group II (included rats that received $\mathrm{CCL}_{4}$ twice weekly for four weeks) \\ Histological results:}

Examination of the $\mathrm{H}$ and $\mathrm{E}$ stained histological sections of the liver of rats that received $\mathrm{CCL}_{4}$ injections for four weeks, revealed that both stroma and parenchyma were greatly affected. An apparent increase in the thickness of capsule and the connective tissue septa was observed. Multiple vacuoles of different sizes and shapes were detected in the capsule as well as in the thickened septa (Fig. 5).

The parenchyma of the liver appeared distorted. Loss of the usual hepatic architecture was detected. Most of the hepatocytes were vacuolated. They contained multiple, large cytoplasmic vacuoles and deeply stained nuclei. Some cells appeared to contain large cytoplasmic vacuoles with flattened peripheral eccentric nuclei were seen between the hepatocytes. Few liver cells still showed deep acidophilic cytoplasm and deeply stained nuclei. Many areas of mononuclear cellular infiltrate were frequently observed around the portal areas (Figs. 6).
In some animals the lesions were patchy in distribution. Their histological sections showed loss of hepatic lobulation. Many specimens showed hepatocytes regenerative nodules separated by wellformed thick fibrous bands formed by plenty of fibroblast and collagen fibers, the fibrous bands surrounded the nodules along with the blood vessels. Many blood vessels were obviously congested (Fig. 7)

In Masson's trichrome stained sections, there was apparent increase in the collagen fibers in the stroma. The increase in collagen fibers was detected around the central veins and surrounding the structure of the portal area (Fig. 8). In many specimens well-formed fibrotic bands were detected in between regenerative hepatocytes nodules with many collagen fibers content and fibroblast infiltrate. The collagen fibers were also detected around the hepatocytes nodule, in between the hepatocytes and around the blood vessels (Figs. 9). Quantification of these data was performed by morphometric analysis of collagen fiber area percentage and revealed a significant increase of collagen fiber content in the diseased group when compared to the control one $(\mathrm{P}<0.05)$.

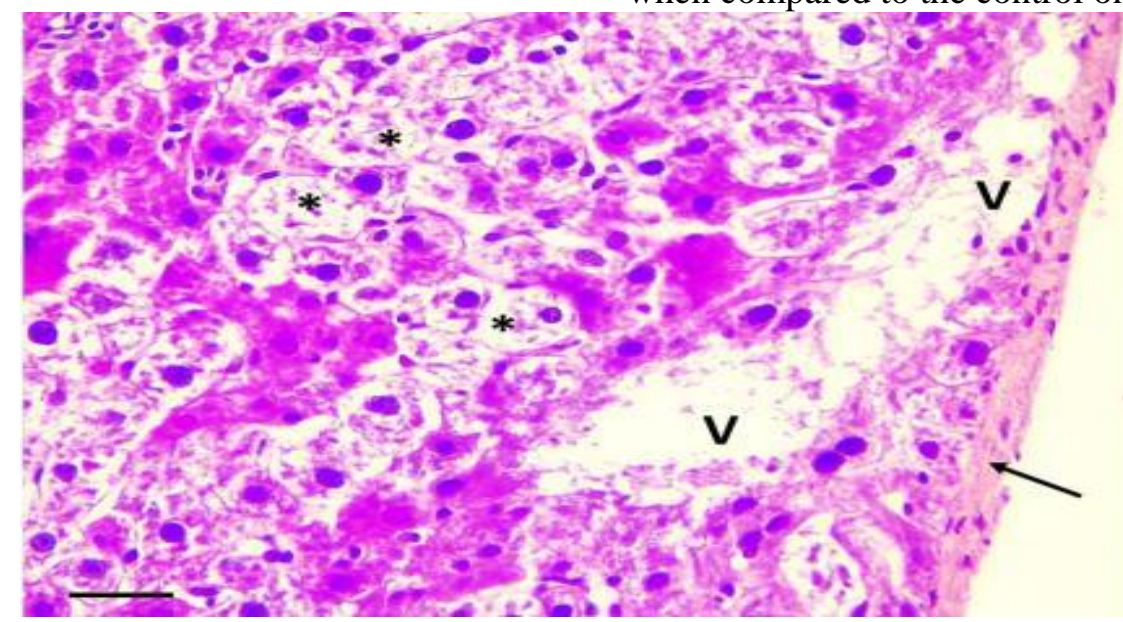

Fig. (5): A photomicrograph of a liver section of a rat of $\mathrm{CCl}_{4}$ group, showing an apparent increase in the thickness of the capsule ( $\uparrow)$. Subcapsular vacuoles of variable sizes and shapes are seen (V). Many hepatocytes showed vacuolated cytoplasm (*). H and E X400 


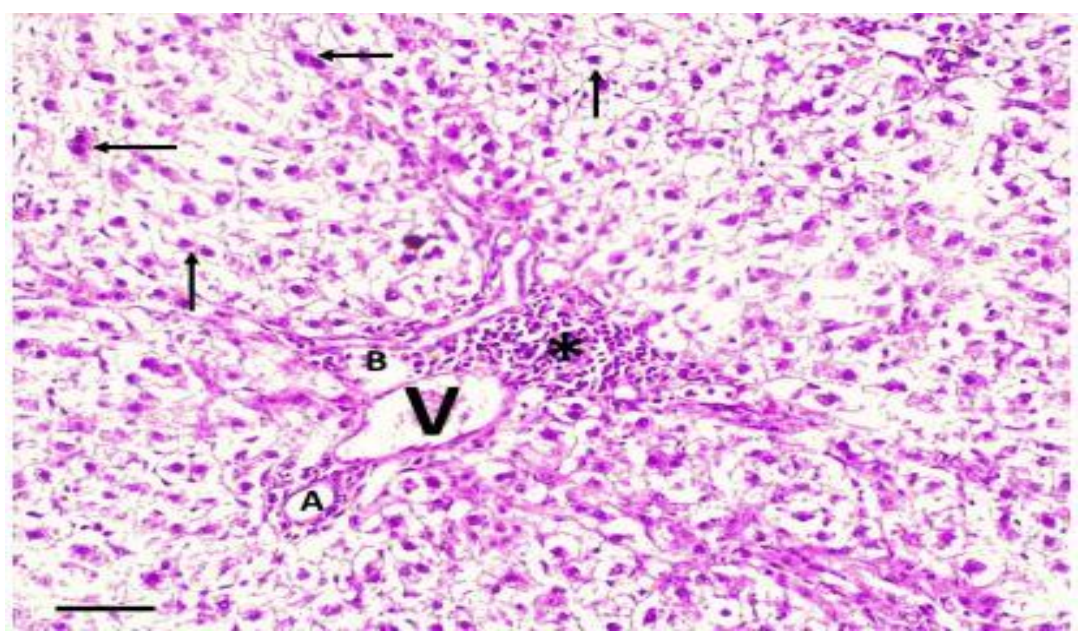

Fig. (6): A photomicrograph of a liver section of a rat of $\mathrm{CCl}_{4}$ group, showing that most of the hepatocytes appear with multiple cytoplasmic vacuoles $(\uparrow)$. Few hepatocytes have deep acidophilic cytoplasm and darkly stained nuclei $(\leftarrow)$. Notice the portal area containing branches of the portal vein $\{\mathrm{V})$, hepatic artery (A) and bile duct (B) surrounded by mononuclear cellular infiltrate (*). H and E X200

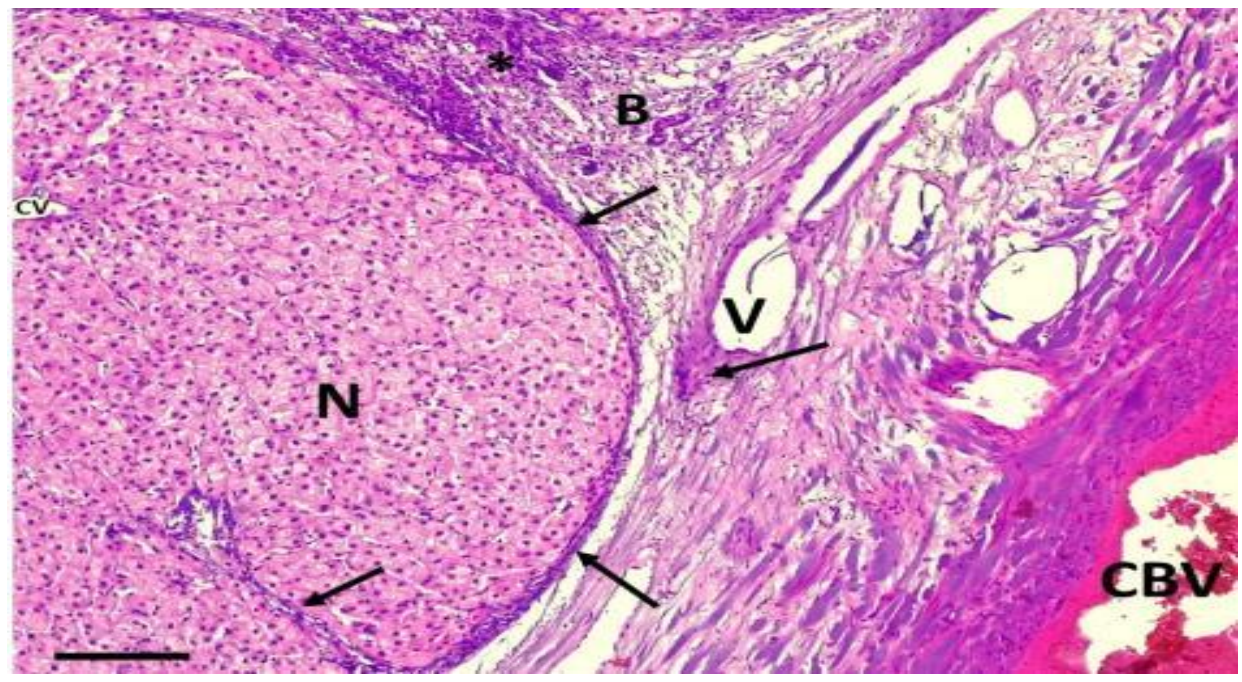

Fig. (7): A photomicrograph of a liver section of a rat of $\mathrm{CCl}_{4}$ group, showing thick fibrous bands (B) formed of many fibroblast $(*)$ and collagen fibers (arrow). The bands intervene between the regenerative hepatocytes nodules (N) and extent around the blood vessels (v). Some blood vessels appeared congested (CBV). H and E X200

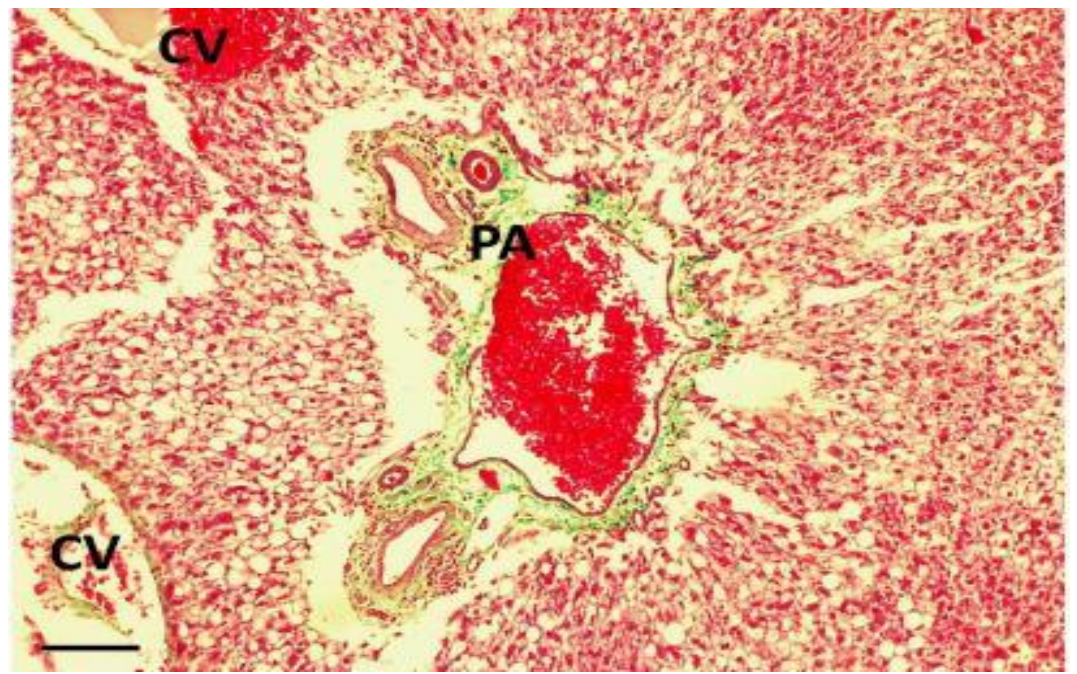

Fig. (8): A photomicrograph of a liver section of a rat of $\mathrm{CCl}_{4}$ group, showing an apparent increase of the collagen fibers in the connective tissue that surround the portal area (PA) and the central vein (CV) Masson's trichrome stain X200 


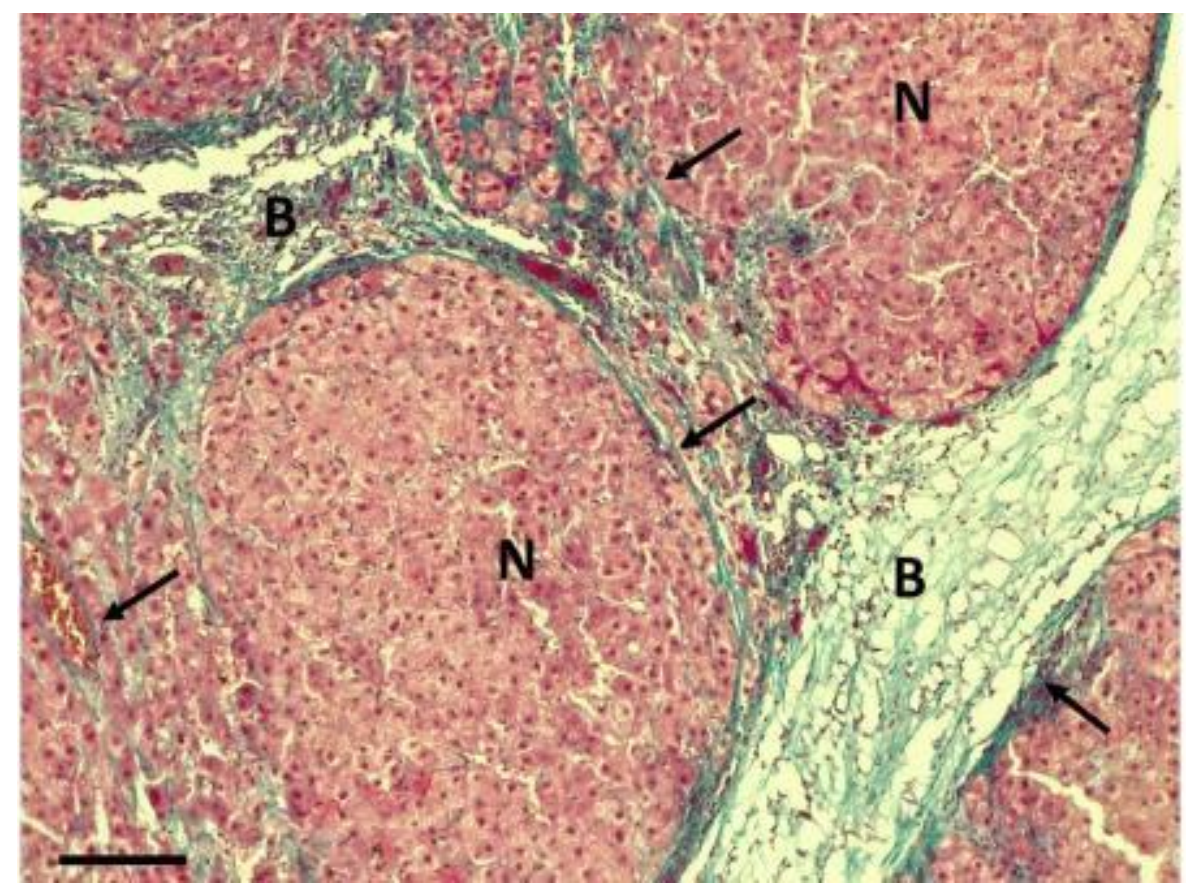

Fig. (9): Photomicrographs of a liver section of a rat of $\mathrm{CCl}_{4}$ group showing well-formed fibrotic bands (B) and abundant collagen fibers $(\uparrow)$ around the regenerative hepatocytes nodules $(\mathrm{N})$ and in-between the hepatocytes. Masson's trichrome stain X200

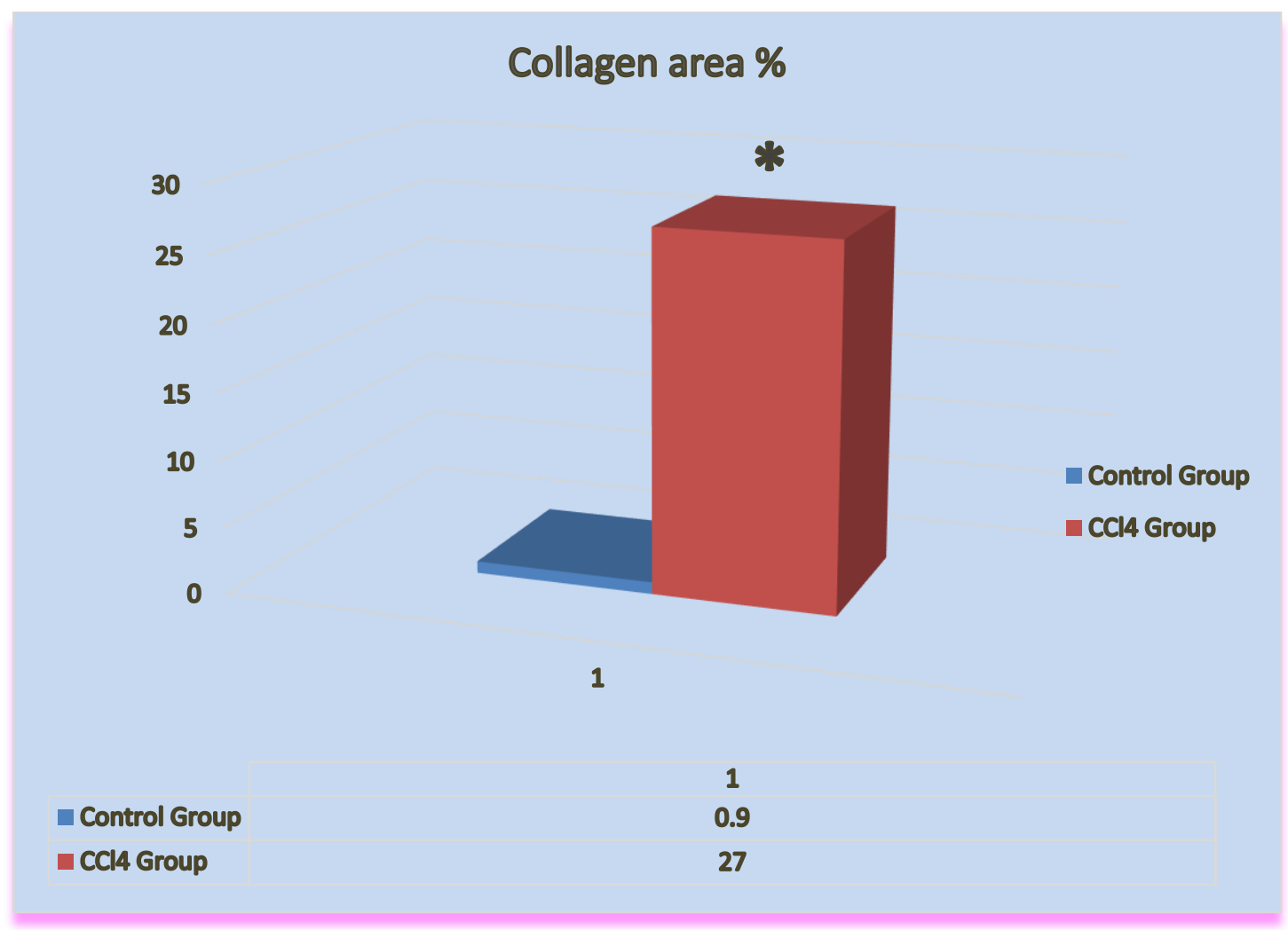

Fig. 10: Comparison of collagen area percentage in the studied groups.

*: $\mathrm{P}<0-05$ i.e. the difference is significant between the two groups. 


\section{DISCUSSION}

Höhme et al. ${ }^{(12)}$ reported that $\mathrm{CCL}_{4}$ initially induced cell death of a pericentral ring of hepatocytes followed by destruction of the characteristic microarchitecture of the hepatic lobules. UskokovićMarković et al. ${ }^{(13)}$ mentioned that the induced liver necrosis by $\mathrm{CCL}_{4}$ is an example of a model for experimental liver necrosis and cirrhosis caused by oxygen free radicals.

$\mathrm{CCL}_{4}$ induced hepatic injury is a very classic model widely used for hepatoprotective drug screening. The acute hepatotoxicity of $\mathrm{CCL}_{4}$ lies in its biotransformation to trichloromethyl free radical $\left(\mathrm{CCL}_{3}\right)$ or trichloroperoxyl radical $\left(\mathrm{CCL}_{3} \mathrm{O}_{2}{ }^{-}\right)$ produced by the mixed-function cytochrome $\mathrm{P} 450$ (CYP) oxygenase system of the endoplasmic reticulum, which causes oxidative stress and membrane damage ${ }^{(7)}$.

Ijiri et al. ${ }^{(14)}$ concluded that $\mathrm{CCL}_{4}$ facilitates the generation of hepatotoxins that can result in morphologic abnormalities and these abnormalities are reasonably characteristic and reproducible for each particular toxin. They also stated that that TNF- $\alpha$ may participate in $\mathrm{CCL}_{4}$-induced liver injury. Li et al. ${ }^{(15)}$ mentioned that $\mathrm{CCL}_{4}$ is commonly used as a model toxicant to induce chronic and acute liver injuries. Acute liver injury was successfully induced by $\mathrm{CCL}_{4}$ as revealed by histopathological results and significant increase in ALT and AST. CCL 4 also caused a decrease in some of the amino acids.

Geerts et al. ${ }^{(16)}$ demonstrated the changes in the liver after four weeks of $\mathrm{CCL}_{4}$ administration. They observed necrotic changes in the centrilobular area with swelling of the hepatocytes and infiltration of ceroid pigment-laden macrophages were seen. The hepatocytes showed enlarged nuclei and increased numbers of hepatic stellate cells (HSCs) which appeared in the area around the central vein. The reticulin and Sirius Red staining demonstrated fibrotic changes in the centrilobular area.

Friedman (17) explained the pathway of HSCs activation and resolution. It is comprised of several discrete responses including proliferation, contractility, fibrogenesis, matrix degradation, retinoid loss and inflammatory cell infiltration. Resolution of fibrosis referred to pathways that either derived the stellate cell to apoptosis, or contribute to their reversion to a more quiescent phenotype.

Vanheule et al. (18) demonstrated the presence of activated HSCs during the development of cirrhosis which appeared in the pericentral area (zone 3 ) and the periportal area (zone 1) where fibrosis first occurred.

Nunes de Carvalho et al. ${ }^{(19)}$ mentioned that liver fibrosis results from chronic injury followed by activation of macrophages and fibrogenic cells like myofibroblasts and activated hepatic stellate cells
HSCs. These fibrogenic cells express alpha smooth muscle actin $\alpha$-SMA and produce extracellular matrix (ECM), with disorganization and loss of function of hepatic parenchyma. It is known that increased levels of matrix metalloproteinase (MMPs) in liver fibrosis are associated with reduction of the pathologic ECM and fibrosis resolution.

Ding et al. (20) induced liver fibrosis by injecting $\mathrm{CCL}_{4}$ IP in rats for eight weeks. They observed disruption of tissue architecture, large fibrous septa formation, pseudolobe separation and collagen accumulation. Electron microscopic examination indicated distorted cell organoides. These changes were accompanied by increase of the levels of alanine aminotransferase (ALT) and aspartate aminotransferase (AST), while albumin was decreased significantly.

Seo et al. (21) stated that fibrosis was a common end stage for a variety of liver diseases, including most chronic liver diseases and results from an imbalance between collagen deposition and degradation.

Sahreen et al. ${ }^{(22)}$ and Veidal et al. (23) reported that the liver is a target organ for the $\mathrm{CCL}_{4}$ toxicity due to its detoxifying function in protecting the body. $\mathrm{CCL}_{4}$ is a well-known hepatotoxic industrial solvent so it is used in diverse experimental models.

Liver fibrosis was induced by $\mathrm{CCL}_{4}$ in adult male albino rats in a study done by Ahmed et al. ${ }^{(24)}$. They mentioned that $\mathrm{CCL}_{4}$ increased serum liver enzymes (ALT, AST, and alkaline phosphatase, lactate dehydrogenase, level of nitric oxide, tumor necrosis factor alpha (TNF $\alpha)$ and liver malondialdehyde content, collagen fiber percent and decreased liver reduced glutathione content as endogenous antioxidant. They added that histopathological changes induced by $\mathrm{CCL}_{4}$ include regenerative nodules, deteriorated parenchyma, and the lobules were infiltrated with fat and structurally altered.

\section{CONCLUSION}

The induced liver fibrosis by $\mathrm{CCL}_{4}$ in rats showed markedly pathological effects on hepatic stroma and parenchyma.

\section{REFERENCES}

1. Doherty $\mathbf{R}$ (2000): A history of the production and use of carbon tetrachloride, tetrachloroethylene, trichloroethylene and 1, 1,1-trichloroethane in the United States: Part 1-Historical background; carbon tetrachloride and tetrachloroethylene. J Environ Forensics, 1: 69-81.

2. Adaramoye O (2009): Comparative effects of vitamin $E$ and kolaviron (a biflavonoid from Garcinia kola) on carbon tetrachloride-induced renal oxidative damage in mice. Pakistan Journal of Biological Sciences, 12:11461151. 
3. Liu F, Liu Z, Wu N, Cong X, Fei R, Chen H, Wei L (2009): Transplanted Endothelial Progenitor Cells Ameliorate Carbon Tetrachloride-Induced Liver Cirrhosis in Rats. Liver Transplantation, 15:1092-1100.

4. El-Kholy A, Hassanen $N$ and Abbas $H$ (2013): Protection of the Mushroom (shiitake "Lentinus-edodes) against Carbon-Tetrachloride-Induced Renal Injury in Rats. Life Science Journal, 10 (1): 235-41.

5. Weber L, Boll M and Stampfl A (2003): Hepatotoxicity and mechanism of action of haloalkanes: Carbon tetrachloride as a toxicological model. Crit. Rev. Toxicol., 33: 105-136.

6. Masuda Y (2006): Learning toxicology from carbon tetrachloride-induced hepatotoxicity. Yakugaku Zasshi., 126 (10): 885-99.

7. Huang H, Wang Y, Zhang Q, Liu B, Wang F, Li J and Zhu R (2012): Hepatoprotective effects of baicalein against $\mathrm{CCl} 4$ - induced acute liver injury in mice. World $\mathbf{J}$ Gastroenterol., 18(45):6605-13.

8. Iredale $J$, Benyon $\mathbf{R}$, Pickering $J$, McCullen M, Northrop M, Pawley S, Hovell C, Arthur MJ (1998): Mechanisms of spontaneous resolution of rat liver fibrosis. Hepatic stellate cell apoptosis and reduced hepatic expression of metalloproteinase inhibitors. J Clin Invest., 102:538-54.

9. Bataller $\mathbf{R}$ and Brenner D (2005): Science in medicine Liver fibrosis. The Journal of Clinical Investigation, 115 (2): 209-218.

10. Guyot C, Lepreux S, Combe C, Doudnikoff E, Bioulac-Sage $P$, Balabaud $C$ and Desmoulière $A$ (2006): hepatic fibrosis and cirrhosis: the myofibroblastic cell subpopulation involved. Int J Biochem Cell Biol., 38: 135-151.

11.Abdel Aziz M, Atta H, Mahfouz S, Fouad H, Roshdy N, Ahmed H, Rashed L, Sabry D, Hassouna A (2007): Therapeutic potential of bone marrow-derived mesenchymal stem cells on experimental liver fibrosis. Clinical Biochemistry, 40: 893-899.

12. Höhme S, Hengstler J, Brulport M, Schäfer M, Bauer A, Gebhardt $R$ and Drasdo D (2007): Mathematical modeling of liver regeneration after intoxication with CCL4. Chem Biol Interact., 168 (1):74-93.

13. Uskoković-Marković S, Milenković $M$, Topić A, Kotur-Stevuljević J, Stefanović $A$ and AntićStanković J (2007): Protective effects of tungstophosphoric acid and sodium tungstate on chemically induced liver necrosis in Wistar rats. J Pharm Sci., 10(3):340-9.

14. Ijiri Y, Kato R, Sadamatsu M, Takano M, Okada Y, Tanaka K and Hayashi T (2014): Chronological changes in circulating levels of soluble tumor necrosis factor receptors 1 and 2 in rats with carbon tetrachlorideinduced liver injury. Toxicology, 0300-483.

15. Li X, Zhang F, Wang D, Li Z, Qin X and Du G (2014): NMR-based metabonomic and quantitative real-time PCR in the profiling of metabolic changes in carbon tetrachloride-induced rat liver injury. J Pharm Biomed Anal, 15(89):42-9.

16. Geerts A, Vanheule E, Praet M, Van Vlierberghe H, De Vos $M$ and Colle I (2008): Comparison of three research models of portal hypertension in mice: macroscopic, histological and portal pressure evaluation. Int J Exp Path., 89: 251-263.

17.Friedman SL (2008): Hepatic fibrosis: overview. Toxicology, 254(3):120-9.

18. Vanheule E, Geerts A, Van Huysse J, Schelfhout D, Praet M, Van Vlierberghe H, De Vos $M$ and Colle I (2008): An intravital microscopic study of the hepatic microcirculation in cirrhotic mice models: relationship between fibrosis and angiogenesis. Int $\mathbf{J}$ Exp Path., 89:419-432.

19. Nunes de Carvalho S, Helal-Neto E, de Andrade D, Costa Cortez E, Thole A, Barja-Fidalgo $\mathrm{C}$ and de Carvalho L (2013): Bone Marrow Mononuclear Cell Transplantation Increases Metalloproteinase-9 and 13 and Decreases Tissue Inhibitors of Metalloproteinase-1 and 2 Expression in the Liver of Cholestatic Rats. Cells Tissues Organs, 198:139-148

20. Ding J, Yu J, Wang C, Hu W, Li D, Luo Y, Luo H and Yu H (2005): Ginkgo biloba extract alleviates liver fibrosis induced by CCL4 in rats. Liver International, 25: 1224-1232.

21.Seo K, Sohn S, Bhang D, Nam M, Lee H, Youn H (2014): Therapeutic effects of hepatocyte growth factoroverexpressing human umbilical cord blood-derived mesenchymal stem cells on liver fibrosis in rats. Cell Biol Int., 38(1):106-16.

22.Sahreen S, Muhammad $K$ and Khan $A$ (2011): Hepatoprotective effects of methanol extract of Carissa opaca leaves on CCl4-induced damage in rat. BMC Complementary and Alternative Medicine, 11:48-55

23. Veidal S, Karsdal M, Vassiliadis E, Nawrocki A, Larsen M, Nguyen Q, Hägglund P, Luo Y, Zheng Q, Vainer $B$ and Leeming D (2011): MMP mediated degradation of type VI collagen is highly associated with liver fibrosis identification and validation of a novel biochemical marker assay. PloS one, 6 (9): 247-53.

24. Ahmed A, Mahmoud M, Ouf $M$ and El-Fathaah $E$ (2011): Aminoguanidine potentiates the hepatoprotective effect of silymarin in CCL4 treated rats. Analysis of Hepatology, 10 (2): 207-215. 